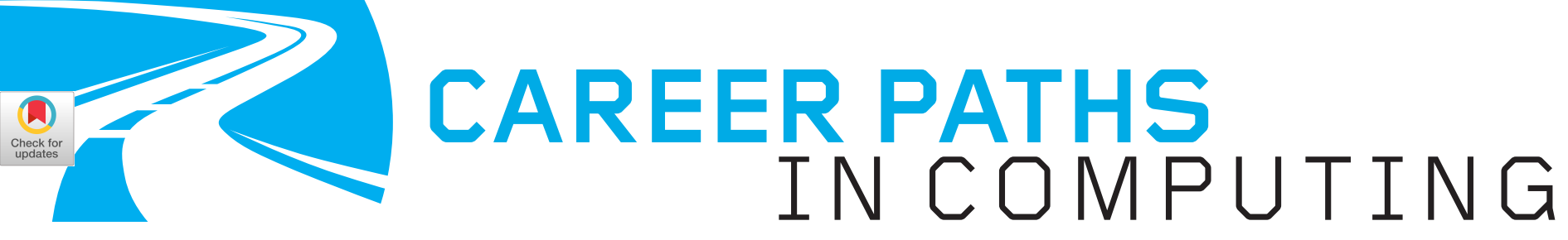

DOI: $10.1145 / 3391913$

\title{
Launching a New Feature in Communications
}

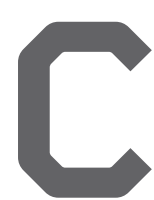

OMPUTING IS AN extraordinarily important transformative technology that has not only grown into a major profession accounting 2.8 million jobs in the U.S. and perhaps four times that number worldwide. Our knowledge of computing technology empowers us, and enables invigorating, varied, and surprising career paths! (see "Computing Is the Secret Ingredient” ).

To celebrate computing's growing importance for society and commerce, increase our understanding of the broad reach of computing, and highlight a diversity of career paths and role models, Communications is launching a new series of one-page articles that will appear prominently in the front of the magazine, under the following charter:

To establish a high-quality, compelling feature that broadens Communications' presentation of the computing profession with a particular focus on a young professional audience and a breadth of career paths. Specifically, to highlight:

- remarkable computing professionals in industry (technology and beyond), government, NGO's, and more. The collection should frame a broad view of contribution, impact, intellectual challenge, and reward - far beyond the academic and research model,

- the global ACM membership, showing career paths in all regions, including Europe, Asia, Oceania, Americas, Middle East, and Africa. In short, a global breadth and inclusive framing of culture, setting, and opportunity, and,

a Chien, A. Computing is the secret ingredient (well, not so secret). Commun. ACM 60, 12 (Dec. 2017), p. 5.
- the exciting range of opportunity and people that computing enables, showcasing a variety of strong role models for young professionals.

In fulfilling this charter, we hope the feature will become a "must read" serial for young computing professionals, inspiring their passion and cultivating an expansive view of contribution, impact, and possibility.

Finally, I'd like to add a personal thanks to Mei Kobayashi for her energy, advocacy, and hard work to help define and launch this new feature!

\section{Andrew A. Chien, EDITOR-IN-CHIEF}

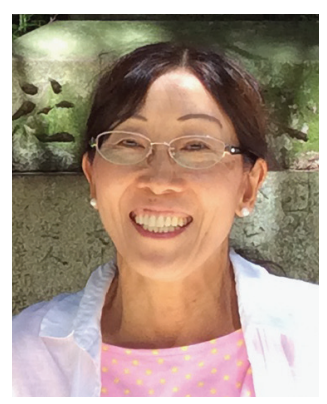

NAME

Mei Kobayashi

BACKGROUND

Born in Tokyo, grew up in

Berkeley, CA, USA

CURRENT JOB TITLE/EMPLOYER

Manager, Customer Services, NTT Communications

LAST DEGREE

Ph.D. Applied Mathematics, University of California at Berkeley

Hello! I am a computational chemistturned-applied mathematician, who joined ACM in the latter part of my career as my technical interests shifted toward computer science. I've been serving on Communications' News Board for several years, and recently, I had the opportunity to brainstorm with Editor-inChief Andrew Chien on broadening $\mathrm{Com}^{-}$ munications' coverage to more accurately reflect the demographics of the ACM membership. We agreed that greater attention should be given to work by computing professionals outside of academia (for example, industry, government, NGOs, start-ups). Communications needed to create a new forum to encourage more participation by these members and leverage their expertise. Then, one day it clicked! The result: this new feature, which showcases careers paths of computing professionals. These personal stories will demonstrate the infinite range of possibilities of what one can do with their computing knowledge.

For this inaugural year, we invited scientists who work in large corporations as well as mavericks who have acquired unique skillsets to make innovative products and services while zig-zagging through a variety of roles. At the same time, we sought to balance geographic and demographic diversity in authorship. Despite their vastly different backgrounds, experiences, and lifestyles, all of our guests write with great passion and infectious enthusiasm, making for delightful reads. It is our hope their collective wisdom will encourage students and young scientists to realize their full potential in a job that also brings joy and fulfilment. As with all new endeavors, we appreciate constructive feedback. Thank you. 